\title{
DIVERSIDAD DE AVES EN BOSQUES DE PINO-ENCINO DEL CENTRO DE GUERRERO, MÉXICO
}

\author{
R. Carlos Almazán-NÚÑEZ1*, Fernando PUebla-Olivares² \\ y Ángel AlmaZÁN-JuÁREZ3 \\ 1Unidad Académica de Ciencias Agropecuarias y Ambientales, Universidad Autónoma de Guerrero, \\ Periférico Poniente s/n, Col. Guadalupe. Iguala, Guerrero, 40030, MÉXICO. \\ 2Instituto Potosino de Investigación Científica y Tecnológica (IPICyT), camino a la presa San José \\ 2055, Col. Lomas 4a sección, C.P. 78216, San Luis Potosí, San Luis Potosí. \\ 3Instituto de Investigación Científica, Área Ciencias Naturales, Universidad Autónoma de Guerrero, \\ Av. Lázaro Cárdenas s/n. Chilpancingo, Guerrero, 39070, MÉXICO \\ *Correspondencia: oikos79@yahoo.com.mx
}

\begin{abstract}
Almazán-Núñez, R. C., F. Puebla-Olivares y A. Almazán-Juárez. 2009. Diversidad de aves en bosques de pino-encino de Guerrero, México. Acta Zool. Mex. (n. s.) 25(1):123-142.

RESUMEN Se estimó la riqueza y abundancia de aves en tres sitios con bosque de pino-encino con diferentes condiciones de desarrollo (bosque maduro, bosque en regeneración y bosque con cafetal) en el centro del estado de Guerrero, México. Entre los tres sitios se registró un total de 117 especies y 1,276 individuos; sin embargo, análisis de curvas de acumulación muestran que es posible registrar más especies para complementar el listado. Se obtuvieron diferencias significativas en la diversidad de especies entre los tres sitios, la mayor diversidad se presentó en el bosque en regeneración, seguido del bosque maduro y por último el bosque con cafetal. La mayor similitud de especies se registró entre el bosque maduro y el bosque en regeneración. No existen diferencias significativas entre los gremios alimentarios de los tres sitios. El estrato medio del bosque fue el más utilizado por las aves en los tres sitios, existiendo diferencias significativas en el uso de los estratos de la vegetación por las aves del bosque en regeneración en comparación con el bosque maduro, así como con el bosque con cafetal.
\end{abstract}

Palabras clave: diversidad de aves, riqueza de especies, abundancia, bosques de pino-encino, Guerrero

Almazán-Núñez, R. C., F. Puebla-Olivares \& A. Almazán-Juárez. 2009. Bird diversity in pine-oak forests from central Guerrero, Mexico. Acta Zool. Mex. (n. s.) 25(1):123-142.

ABSTRACT We assessed bird species richness and their abundances at three sites with pine-oak forest under different development stages (mature forest, regenerating forest and shade coffee plantation) in central Guerrero, Mexico. We recorded a total of 117 species and 1,276 individuals; however, analysis of cumulative species curves indicates that it is possible to find more species in the region for a more complete list. There were significant differences in species diversity among the three sites. Species diversity was higher at the regenerating forest, middle in mature forest and lowest at shade coffee plantation. Higher similarity was between mature forest and regenerating forest. There were no significant differences among the feeding guilds at any site. The mid-stratum had the highest utilization at the three sites. In addition, there were significant differences among strata in regenerating forest in comparison with mature forest and shade coffee plantation.

Key words: avian diversity, species richness, abundance, pine-oak forests, Guerrero 
Almazán-Núñez et al.: Diversidad de aves en bosques de pino-encino de Guerrero

\section{INTRODUCCIÓN}

A pesar de que México se caracteriza por ser un país esencialmente con clima tropical y semiárido, resulta sobresaliente que una gran proporción de su extensión territorial está cubierta por bosques de climas templados como el de pino-encino (Rzedowski 1978, Challenger 1998). Este tipo de vegetación constituye el hábitat para un gran número de especies vegetales y animales, muchas de las cuales se encuentran amenazadas debido principalmente al cambio en el uso del suelo (FloresVillela \& Gérez 1994, Toledo \& Ordóñez 1998). En México como en otros países, este hábitat alberga una gran cantidad de aves (Escalante et al. 1998), algunas de las cuales son endémicas y de gran importancia para la conservación, e. g. Dendrortyx macroura, Aulacorhynchus prasinus, Lepidocolaptes leucogaster, Vireo hypochryseus, Cyanolyca mirabilis, Ergaticus ruber (Howell \& Webb 1995). Por esta razón, es importante caracterizar a escalas locales la riqueza y la diversidad de aves en bosques templados, como el de pino-encino, ya que además es uno de los hábitat más amenazados por la creciente demanda del recurso forestal maderable, así como por los bienes y servicios que éstos ofrecen (Fregoso et al. 2001).

La región central del estado de Guerrero se caracteriza por presentar extensas áreas de bosque de pino-encino; sin embargo, este hábitat está sometido a fuertes presiones antropogénicas en la zona, por lo que su cobertura original se ha ido reduciendo a relictos cada vez más pequeños (Luna \& Llorente 1993). Desde el punto de vista avifaunístico, esta región es probablemente la mejor estudiada dentro de la entidad, ya que existen exploraciones desde finales del siglo XIX que revelan la importancia del bosque de pinoencino como hábitat para numerosas especies de aves (Navarro 1998). Sin embargo, la mayor parte de los estudios que se han llevado a cabo en las zonas montanas de la región central del estado estuvieron enfocados a la descripción y descubrimiento de nuevos taxa (Griscom 1937, Van Rossem 1940, Martín del Campo 1948, Blake 1950, Dixon \& Davis 1958), al registro de presencia de especies (Arnold \& Maxwell 1970, Arnold 1971), o al inventario de avifaunas de ciertas áreas de interés (Navarro \& Muñoz 1990, Navarro \& Escalante 1993). En cambio, los trabajos sobre comunidades de aves que analizan las relaciones entre sus ambientes en términos de diversidad y abundancia son escasos, a pesar de que este tipo de información es de singular importancia para el manejo apropiado de los recursos faunísticos, así como criterio de evaluación de la calidad del hábitat (Ojasti 2000, Balmer 2001), especialmente en áreas sometidas a fuertes procesos de deterioro como es el bosque de pino-encino.

En este trabajo se analiza y se compara la riqueza, diversidad, abundancia de especies, composición de gremios alimentarios y utilización de estratos vegetativos por la avifauna en tres sitios con bosque de pino-encino, con diferentes condiciones de desarrollo (bosque maduro, bosque en regeneración y bosque con cafetal) localizados en el centro del estado de Guerrero, con el fin de apoyar en la toma de decisiones sobre manejo y conservación de las poblaciones de aves en esta región. 


\section{MATERIAL Y MÉTODOS}

Área de estudio. Este estudio se llevó a cabo en tres sitios con bosque de pinoencino con diferentes condiciones localizados en el centro de Guerrero (Fig. 1), en la Sierra Madre del Sur: bosque maduro del Parque Ecológico de Omiltemi (17 30' N, $\left.99^{\circ} 40^{\prime} \mathrm{W}\right)$, bosque en regeneración del ejido Los Morros (17 $\left.41^{\prime} \mathrm{N}, 99^{\circ} 49^{\prime} \mathrm{W}\right)$ y

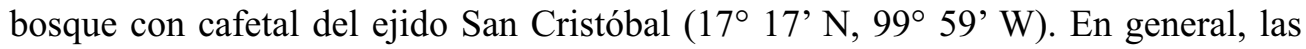
características ambientales en los tres sitios son muy similares, presentando un clima predominantemente templado subhúmedo con lluvias en verano (García 1981). La altitud en cada uno de los sitios es de 2,550 msnm para el bosque maduro, 2,200 msnm para el bosque en regeneración y $1,700 \mathrm{msnm}$ para el bosque con cafetal.

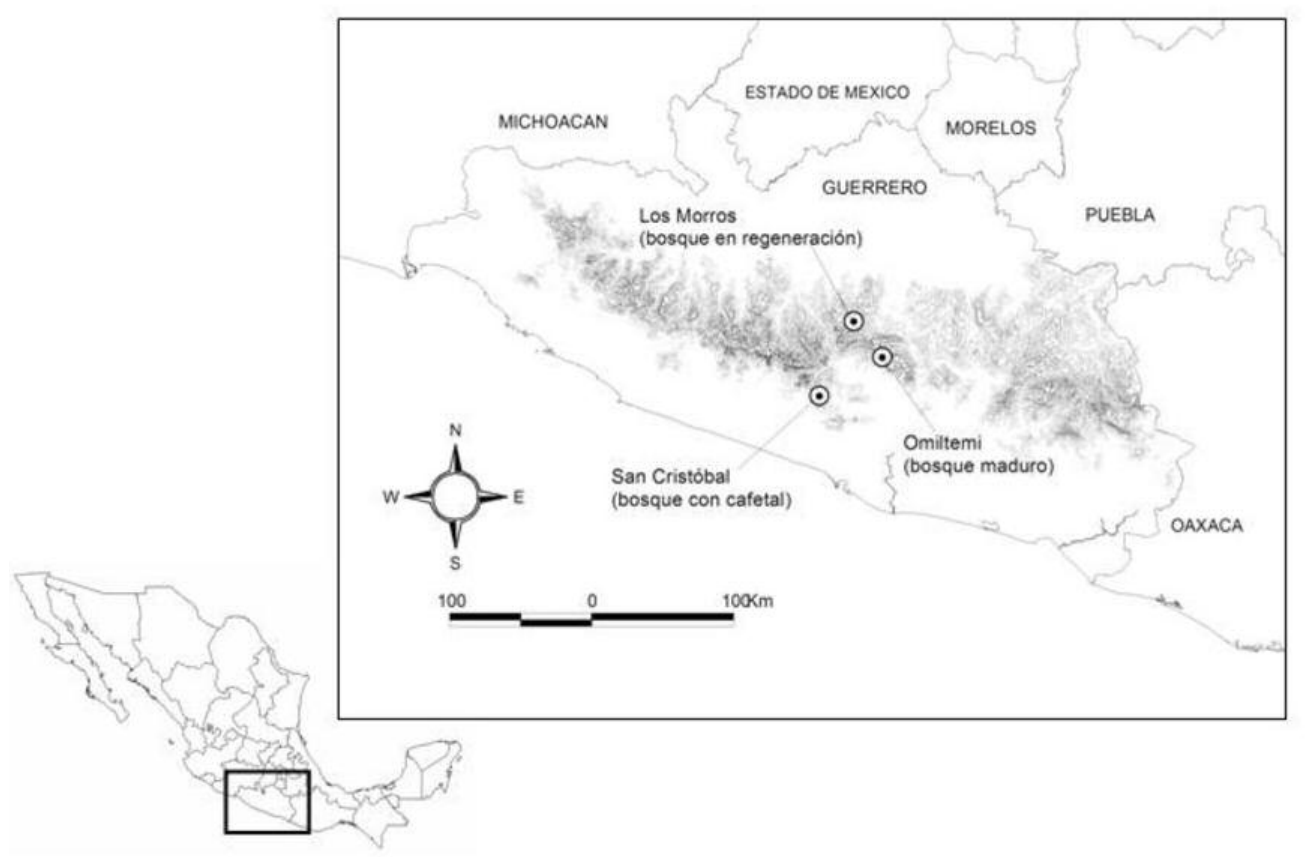

Figura 1. Localización de los sitios de estudio en el centro de Guerrero (Sierra Madre del Sur), México. El mapa topográfico delineado de la Sierra Madre del Sur se obtuvo de CONABIO (http://www.conabio.gob.mx).

El área de bosque maduro se localiza dentro del Parque Ecológico de Omiltemi. Esta zona, si bien no llegó a establecerse como un Área Natural Protegida toda vez que no existe ningún decreto que lo avale, es una de las regiones del país más exploradas, como lo muestran los itinerarios de las Reales Expediciones Científicas hacia finales del siglo XVIII y principios del XIX (Luna \& Llorente 1993). Además, mantiene áreas con buen estado de conservación donde el dosel es cerrado y en 
consecuencia no permite mucha entrada de luz al interior del bosque. Las especies arbóreas predominantes son Pinus lawsonii, P. montezumae, Quercus castanea, $Q$. candicans y $Q$. crassifolia, mientras que en el estrato arbustivo se encuentran Arbutus xalapensis, Penstemon kunthii, Ceanothus coeruleus y Baccharis conferta; en el estrato herbáceo predominan Bouvardia laevis, Fuchsia fulgens y Coreopsis mutica.

En el bosque en regeneración, los árboles están conformados principalmente por individuos jóvenes producto de programas de reforestación implementados en la zona en los últimos años, así como por procesos de regeneración natural. El estrato arbóreo está conformado por Pinus oaxacana, P. pringlei, P. chiapensis, Quercus glaucoides y $Q$. obtusata, en el estrato arbustivo se encuentran Arbutus xalapensis y Alnus jorullensis, y el estrato herbáceo está representado por Dahlia coccinea, Adiantum cocinnum, Salvia lavanduloides y Euphorbia graminea.

En el bosque con cafetal, el estrato arbóreo está representado por Pinus oocarpa, $P$. pringlei, Quercus glaucescens y Q. candicans; el estrato arbustivo por Arbutus xalapensis, Acacia farnesiana y Ardisia compressa, y el herbáceo integrado principalmente por Begonia biserrata, Besera sp. y Varbacum thapus. Dentro de esta zona, existen cafetales, muchos de los cuales se encuentran actualmente abandonados.

Durante los meses de julio, septiembre y octubre de 2003, febrero, julio y septiembre de 2004, y julio y agosto de 2006, se desarrollaron muestreos en los tres sitios de estudio abarcando un total de 32 días de trabajo efectivo de campo, con un promedio de diez días en cada sitio. Se obtuvieron registros visuales y auditivos de las especies a lo largo de transectos lineales de aproximadamente $2 \mathrm{~km}$ de longitud (Emlen 1971), los cuales se recorrieron diariamente durante las mañanas sobre algunas veredas existentes. Adicionalmente, se realizaron capturas de ejemplares utilizando ocho redes de niebla de 12 metros de longitud por 2.10 de alto y un tamaño de malla de $25 \mathrm{~mm}$, las cuales estuvieron extendidas de las 6:30 a las 18:00 horas, con un total de 2,360 horas-red. La determinación taxonómica de los especimenes colectados y observados se realizó mediante las guías de campo de Peterson y Chalif (1989), Howell y Webb (1995) y National Geographic Society (1999). La nomenclatura y el arreglo sistemático de las especies está basado en la propuesta de la American Ornithologists' Union (1998).

Para cada especie se determinó su estatus estacional de acuerdo a Howell y Webb (1995): residente permanente, migratoria de invierno, migratoria de verano y transitoria. El endemismo se determinó con base en Navarro y Benítez (1993) y González-García y Gómez de Silva (2003). El estatus de conservación se basó en la NOM-059-SEMARNAT-2001 (DOF 2002). La abundancia relativa de las especies se basó en las categorías de Pettingill (1969): abundante (más de 16 individuos registrados diariamente), común (11 a 15 individuos), moderadamente común (7 a 10 
individuos), poco común (4 a 6 individuos) y rara (1 a 3 individuos). Los gremios tróficos fueron asignados de acuerdo con la dieta de cada especie, determinada con base en observaciones personales y datos bibliográficos (Coates-Estrada \& Estrada 1985, Ehrlich et al. 1988, Arizmendi et al. 1990, Puebla-Olivares 2001, Stiles et al. 2003): insectívoros, frugívoros, nectarívoros, granívoros, depredadores, omnívoros y carroñeros, considerando también a aquellas especies con dietas mixtas (e.g. insectívoro-frugívoro). Para determinar el uso de estratos vegetativos en cada sitio, se contabilizó el número total de individuos observados de cada especie en cada estrato a lo largo de los transectos, clasificándolos en estrato inferior (suelo y herbáceo), estrato medio (arbustivo y dosel bajo) y estrato superior (dosel alto), por ser este el modo frecuente de observarlas.

Análisis de datos. Para los tres sitios en conjunto y por separado se estimó la riqueza total esperada con base en modelos no paramétricos basados en incidencia: Chao 2, Jacknife de segundo orden, estimador de cobertura basado en incidencia (ICE) y Bootstrap (Chazdon et al. 1998), utilizando el programa EstimateS v. 8 (Colwell 2006). Estos modelos fueron comparados con el objeto de evaluar su desempeño y determinar cual de éstos se ajustaba mejor a los datos. Para eliminar la influencia del orden en el cual los días de muestreo fueron agregados al total de muestras, se aleatorizó el orden de las muestras 100 veces (Colwell \& Coddington 1994). Este procedimiento suaviza la curva de acumulación de especies mediante reordenamiento aleatorio repetido (100 repeticiones) de las muestras (Longino y Colwell 1997). Asimismo, como criterio adicional para evaluar la representatividad de la lista de especies se utilizó el propuesto por Gómez de Silva y Medellín (2001), el cual consiste en reconocer listas incompletas cuando faltan taxa "omnipresentes", y si contienen un cierto número mínimo de especies y familias (menos de 35 especies y 21 familias).

La diversidad y la equidad (E) para cada sitio se determinaron a partir de los datos de riqueza y abundancia utilizando el índice de Shannon-Wiener $\left(H^{\prime}\right)$ y el de Simpson $(\lambda)$. Las diferencias en la diversidad de especies fueron evaluadas utilizando el índice de diversidad de Shannon-Wiener y una prueba de $t$ de Student (Magurran 1989, Brower et al. 1998). Además, para obtener una idea más clara de las especies efectivamente presentes o importantes en cada hábitat, se utilizaron los "Números de diversidad de Hill", los cuales transforman los valores de los índices de diversidad de Shannon-Wiener $\left(H^{\prime}\right)$ y de Simpson $(\lambda)$ en "unidades de número de especies" combinando la riqueza específica y la equitatividad de las mismas (Ludwig y Reynolds 1988). Estos números de diversidad son el Número 1 de Hill: $\mathrm{N} 1=e^{H^{\prime}}$ donde $e$ es la base del logaritmo natural y $H^{\prime}$ es el índice de diversidad de ShannonWiener; y Número 2 de Hill: N2=1/ $\lambda$, donde $\lambda$ es el índice de diversidad de Simpson (Ludwig \& Reynolds 1988). Los números de Hill permiten ponderar cada especie por su proporción y al incrementarse ésta se le da menos peso a las especies raras y se 
obtienen valores más bajos de estos índices; su valor es máximo cuando todas las especies contribuyen con las mismas abundancias y mínimo cuando pocas especies contribuyen con las mayores abundancias, tomando un intervalo de 1 a $n$ donde $n$ es el número total de especies. Se considera que N1 es el número de especies abundantes y N2 es el número de especies muy abundantes en la muestra (Ludwig y Reynolds 1988).

Para la determinación de la similitud avifaunística entre los sitios se empleó el Coeficiente de Jaccard, el cual es ampliamente usado para comparar asociaciones entre datos de presencia-ausencia (Moreno 2001). Para comparar la proporción entre los gremios alimentarios de los tres sitios, así como la utilización de estratos vegetativos se utilizó una prueba de $X^{2}$. Todos los análisis fueron considerados estadísticamente significativos cuando $\mathrm{P}<0.05$.

\section{RESULTADOS}

Se registró un total de 117 especies de aves entre los tres sitios agrupadas en 92 géneros, 35 familias y 13 órdenes (Apéndice 1). La familia Parulidae presentó la mayor riqueza de especies (19 especies), seguida por Tyrannidae (10 especies) y Emberizidae (8 especies). Del total de especies, 99 son residentes permanentes, 17 son migratorias de invierno y una es migratoria de verano. Además, un total de 14 especies son endémicas a México (Streptoprocne semicollaris, Eupherusa poliocerca, Piculus auricularis, Vireo brevipennis, Cyanolyca mirabilis, Thryothorus sinaloa, T. felix, Catharus occidentalis, Melanotis caerulescens, Ergaticus ruber, Piranga erythrocephala, Atlapetes pileatus, Melozone kieneri, Pipilo ocai) y seis cuasiendémicas (Momotus mexicanus, Empidonax affinis, Vireolanius melitophrys, Ptilogonys cinereus, Junco phaeonotus, Icterus graduacauda). Entre las especies registradas, existen especies exclusivas a la provincia biótica de la Sierra Madre del Sur como Eupherusa poliocerca y Cyanolyca mirabilis (Apéndice 1). Por otro lado, 16 especies están dentro de alguna categoría de riesgo según la NOM-059SEMARNAT-2001 (DOF 2002); diez están sujetas a protección especial (Accipiter striatus, A. cooperii, Aratinga canicularis, Streptoprocne semicollaris, Trogon collaris, Aulacorhynchus prasinus, Dendrocolaptes sanctithomae, Myadestes occidentalis, Melanotis caerulescens, Vermivora crissalis), cuatro amenazadas (Geotrygon albifacies, Automolus rubiginosus, Catharus frantzii, Oporornis tolmiei) y dos en peligro de extinción (Eupherusa poliocerca, Cyanolyca mirabilis).

Los modelos no paramétricos utilizados para construir las curvas de acumulación para los tres sitios en conjunto sugieren que aún faltan más especies por registrarse (Fig. 2). Los modelos ICE y Chao 2 presentan valores estimados muy similares (143 y 146 especies respectivamente), mientras que Jacknife 2 sugiere un total de 166 especies y Bootstrap 131 especies. Los valores estimados por cada sitio se muestran en el Cuadro 1. 


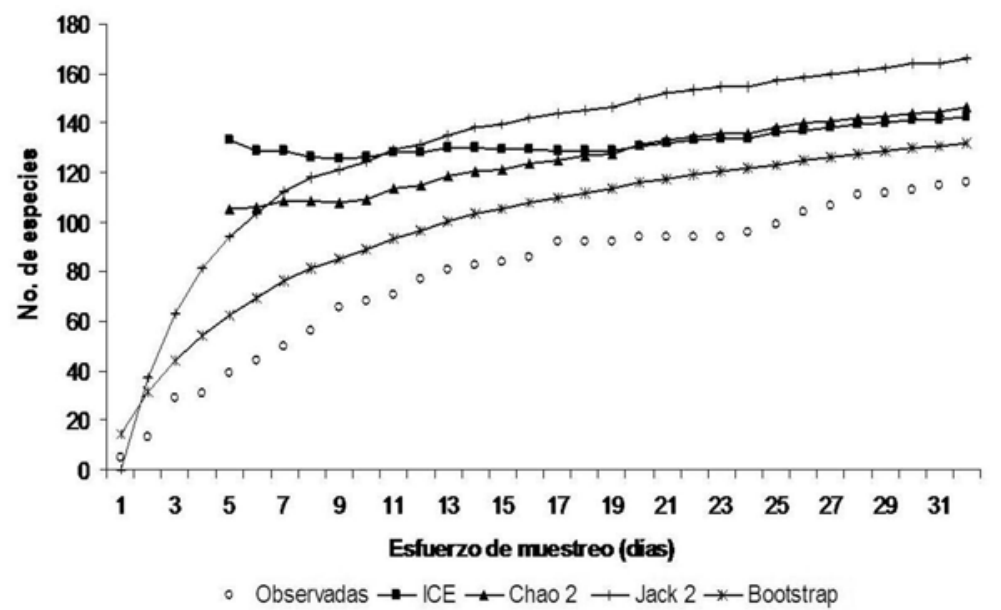

Figura 2. Curva de acumulación de especies y estimadores no paramétricos utilizados para los tres sitios en conjunto. Las estimaciones de los modelos ICE y Chao 2 de los cuatro primeros días no se incluyeron debido a que fueron valores muy altos.

Cuadro 1. Valores estimados de los modelos no paramétricos utilizados en cada sitio de estudio y su desviación estándar (entre paréntesis).

\begin{tabular}{lccccc}
\hline Sitios de estudio & Especies & \multicolumn{4}{c}{ Estimadores de riqueza } \\
& & ICE & Chao 2 & Jack 2 & Bootstrap \\
\hline Bosque maduro & Observadas & 65 & 65 & 65 & 65 \\
& Estimadas & $84(4.82)$ & $78(5.0)$ & $91(19.76)$ & $74(17.20)$ \\
\multirow{2}{*}{ Bosque en regeneración } & Observadas & 71 & 71 & 71 & 71 \\
& Estimadas & $101(20.61)$ & $97(15.0)$ & $112(32.70)$ & $83(20.62)$ \\
\multirow{2}{*}{ Bosque con cafetal } & Observadas & 40 & 40 & 40 & 40 \\
& Estimadas & $50(3.25)$ & $48(2.55)$ & $56(18.44)$ & $45(10.21)$ \\
\hline
\end{tabular}

El análisis comparativo de la riqueza entre los tres sitios con bosque de pinoencino, indicó que la mayor riqueza y abundancia se presentó en el bosque en regeneración con 71 especies y 496 individuos. La diversidad estimada para este sitio fue de $\mathrm{H}^{\prime}=3.97, \lambda=0.023$ y $\mathrm{E}=0.93$; estos valores transformados en los números de diversidad de Hill, N1 y N2 mostraron que 53 y 42 especies son abundantes o muy abundantes respectivamente en este tipo de vegetación y contribuyen efectivamente a la diversidad del mismo con abundancias relativas que van de 24 a 3 individuos, mientras que las especies restantes mostraron una abundancia relativa de 2 a 1 individuo registrados durante el trabajo de campo (Apéndice 1). El número de especies abundantes o muy abundantes estimado con los números de Hill y que 
contribuyen efectivamente a la diversidad en este sitio coinciden aproximadamente con las especies incluidas en las categorías de Pettingill (1969), de abundantes (ocho especies), comunes (ocho especies), moderadamente comunes (12 especies), poco comunes (16 especies) y que en total suman 44 especies, mientras que las restantes 27 especies son raras con registros de 1-3 individuos. Las especies abundantes fueron: Amazilia beryllina, Basileuterus rufifrons, Myioborus pictus, Myadestes occidentalis, Columbina inca, Junco phaeonotus, Cathartes aura y Melanerpes formicivorus (Apéndice 1).

En el bosque maduro se registraron 65 especies y 482 individuos. El índice de diversidad para este bosque fue de $\mathrm{H}^{\prime}=3.80, \lambda=0.028$ y $\mathrm{E}=0.91$, mientras que $\mathrm{N} 1$ y N2 mostraron 45 y 35 especies abundantes o muy abundantes respectivamente y que contribuyen de manera efectiva a la diversidad de especies en el bosque maduro con abundancias relativas de 30 a 4 individuos, mientras que las especies restantes presentan abundancias relativas de 3 a 1 individuo (Apéndice 1). De acuerdo con las categorías de Pettingill (1969), ocho especies son abundantes, ocho comunes, siete moderadamente comunes, 20 poco comunes y 22 raras. Las especies más abundantes en este sitio fueron: Buarremon brunneinucha, Basileuterus belli, Chlorospingus ophthalmicus, Myadestes occidentalis, Stelgidopteryx serripennis, Myioborus miniatus, Henicorinha leucophrys y Amazilia beryllina (Apéndice 1).

Por último, en el bosque con cafetal se registraron 40 especies y 298 individuos. El índice de diversidad para este bosque fue de $\mathrm{H}^{\prime}=3.34, \lambda=0.043$ y $\mathrm{E}=0.90$. Por otra parte N1 indicó que 28 especies son abundantes y N2 mostró que 23 especies son muy abundantes contribuyendo de manera efectiva a la diversidad en el bosque con cafetal con abundancias relativas de 28 a 4 individuos, mientras que las especies restantes mostraron abundancias relativas de 3 a 1 individuos (Apéndice 1). De acuerdo a las categorías de Pettingill (1969), en este tipo de vegetación un total de cinco especies fueron abundantes, siete comunes, cinco moderadamente comunes, ocho poco comunes y 15 raras, siendo las especies abundantes: Myadestes occidentalis, Aratinga canicularis, Buarremon brunneinucha, Basileuterus culicivorus y Chlorospingus ophthalmicus (Apéndice 1).

Existen diferencias significativas entre la diversidad de especies de los tres sitios de bosque de pino-encino (regeneración $v s$ maduro: $\mathrm{t}_{1569(2)}=3.64, \mathrm{P}<0.05$; regeneración $v s$ cafetal: $\mathrm{t}_{718(2)}=12.17, \mathrm{P}<0.05$; maduro $v s$ cafetal: $\mathrm{t}_{262(2)}=8.55 \mathrm{P}$ $<0.05)$. De acuerdo con el índice de Jaccard, el bosque en regeneración tiene una similitud de $41.66 \%$ con el bosque maduro, y $16.84 \%$ con el bosque con cafetal; por último, las áreas de bosque maduro y con cafetal presentan una similitud de $19.31 \%$.

Del total de especies registradas, 14 se distribuyen en los tres sitios y 31 se registraron en dos sitios (Apéndice 1). El número de especies exclusivas en un sólo sitio fue mayor en el bosque en regeneración con 29, predominando especies más generalistas (e.g. Crypturellus cinnamomeus, Vireolanius melitophrys, Cyanocitta 
stelleri, Aimophila rufescens), seguido del bosque maduro con 22, donde se registraron especies especialistas de bosques conservados (e.g. Vireo brevipennis, Cyanolyca mirabilis, Basileuterus belli, Pipilo ocai), y el bosque con cafetal con 21 (e.g. Geotrygon albifacies, Phaetornis longirostris, Eupherusa poliocerca, Lamprolaima rhami, Dendrocolaptes sanctithomae).

Con respecto a los gremios alimentarios de las especies, se obtuvo que los insectívoros estuvieron mejor representados en el área de estudio (55 especies), seguido de los omnívoros (13 especies), granívoros (8 especies) y depredadores (7 especies), así como también dentro de las especies con dietas mixtas, los insectívorofrugívoros fueron los mejor representados (17 especies) (Cuadro 2). En los tres sitios de estudio, el gremio más importante fue también el de los insectívoros, aunque el orden de los demás gremios no fue exactamente el mismo (Cuadro 2). Al analizar comparativamente los gremios alimentarios, se encontró que no hay diferencias significativas entre los tres sitios (regeneración vs maduro: $X^{2}=2.86$, g.1. $=7, \mathrm{P}$ $>0.05$; regeneración $v s$ cafetal: $X^{2}=2.47$, g.l. $=7, \mathrm{P}>0.05$; maduro $v s$ cafetal: $X^{2}=$ 2.87, g.1. $=7, \mathrm{P}>0.05)$.

Cuadro 2. Número de especies en el área de estudio y en cada uno de los tres sitios con presencia de bosque de pino-encino de acuerdo al gremio alimentario en el centro de Guerrero, México. Entre paréntesis se indica el porcentaje en la muestra.

\begin{tabular}{lcccc}
\hline \multirow{2}{*}{ Gremio al imentario } & \multicolumn{4}{c}{ Sitios de estudio } \\
\cline { 2 - 5 } & Bosque maduro & $\begin{array}{c}\text { Bosque en } \\
\text { regeneración }\end{array}$ & $\begin{array}{c}\text { Bosque con } \\
\text { cafetal }\end{array}$ & $\begin{array}{c}\text { Área de } \\
\text { estudio }\end{array}$ \\
\hline Carroñero & $2(3.0)$ & $2(2.8)$ & $2(5.0)$ & $2(1.7)$ \\
Depredador & $5(7.7)$ & $3(4.2)$ & $2(5.0)$ & $7(6.0)$ \\
Granívoro & $3(4.6)$ & $5(7.0)$ & $3(7.5)$ & $8(6.8)$ \\
Granívoro-insectívoro & $6(9.2)$ & $5(7.0)$ & $1(2.5)$ & $7(6.0)$ \\
Insectívoro & $27(41.5)$ & $33(46.4)$ & $17(42.5)$ & $55(47.0)$ \\
Insectívoro-frugívoro & $12(18.4)$ & $10(14.0)$ & $7(17.5)$ & $17(14.5)$ \\
Nectarívoro-insectívoro & $5(7.7)$ & $4(5.6)$ & $4(10.0)$ & $8(6.8)$ \\
Omnívoro & $5(7.7)$ & $9(12.6)$ & $4(10.0)$ & $13(11.1)$ \\
& & & & $117(100)$ \\
\hline
\end{tabular}

El estrato más utilizado por las aves en los tres sitios fue el estrato medio del bosque maduro con 192 individuos, 174 en el bosque en regeneración y 99 en el bosque con cafetal. En el bosque en regeneración el segundo estrato en importancia fue el estrato inferior con 166 individuos, en cambio para el bosque maduro y el 
bosque con cafetal el estrato superior siguió en importancia con 113 y 89 individuos, respectivamente. De manera contraria, en el bosque en regeneración el estrato superior fue el que menos individuos presentó (94 individuos), y en el bosque maduro y con cafetal el estrato inferior fue el último en importancia (107 y 70 individuos respectivamente). La comparación entre usos de estratos vegetativos reveló diferencias significativas entre las áreas de bosque en regeneración y maduro $\left(X^{2}=14.81\right.$, g.l. $\left.=2, \mathrm{P}<0.05\right)$, así como entre las áreas de bosque en regeneración y con cafetal $\left(X^{2}=16.06\right.$, g.l. $\left.=2, \mathrm{P}<0.05\right)$, mientras que entre el bosque maduro y con cafetal no se observaron diferencias significativas $\left(X^{2}=5.18\right.$, g.1. $\left.=2, \mathrm{P}>0.05\right)$.

\section{DISCUSIÓN}

En este estudio se documentó la presencia de 117 especies de aves, lo que representa el 21.4\% del total registrado para el estado de Guerrero (Navarro 1998). La información sobre la relación entre los bosques de pino-encino y la diversidad de aves, entre otros atributos ecológicos medidos en este trabajo es de singular importancia, ya que bajo estas características pocos son los trabajos que se han desarrollado en los bosques templados mixtos de Guerrero. Además, la riqueza de especies obtenida es importante en el conocimiento de la avifauna del bosque de pino-encino de la región central del estado, ya que previamente sólo existía un inventario relativamente completo del parque ecológico de Omiltemi (Navarro \& Escalante 1993), región que históricamente ha sido la mejor estudiada y ha significado la localidad tipo de una gran cantidad de taxa, tanto para aves (Van Rossem 1940, Miller \& Storer 1950), como para otros grupos taxonómicos (Luna \& Llorente 1993).

Al comparar el inventario obtenido en este estudio con el bosque de pino-encino del parque ecológico de Omiltemi, donde se han registrado 114 especies de aves de las 160 registradas para el parque en su totalidad (Navarro \& Escalante 1993), se observa que 65 especies se encuentran compartidas entre ambas zonas. Muchas de las especies que no fueron registradas en este estudio, aunque se infiere su distribución potencial en esta región (sensu Howell \& Webb 1995), incluyen especies de difícil identificación como las de hábitos altamente aéreos (Cypseloides rutilus, Streptoprocne zonaris), de hábitos nocturnos (Asio stygius, Megascops trichopsis), migratorias de invierno (Selasphorus rufus, Melospiza lincolnii), así como una gran cantidad de especies de las cuales sólo existen registros históricos, pero no actuales, lo cual sugiere que muchas de ellas podrían estar localmente extintas en la zona (e.g. Ara militaris, Turdus infuscatus).

Las especies anteriormente mencionadas complementaría el inventario obtenido para la región, ya que de acuerdo con las curvas de acumulación, los valores estimados tanto para la zona de estudio como para los tres sitios por separado, muestran que aún faltan más especies por registrarse. En este sentido, el uso de 
estimadores de riqueza de especies es útil para juzgar cuán completa es una lista determinada (Gómez de Silva \& Medellín 2001). Adicionalmente, criterios como el propuesto por Gómez de Silva y Medellín (2001), sugieren que la lista de especies obtenida en este estudio es aproximadamente completa y representativa, ya que se registraron especies en las familias o géneros mencionados por estos autores, así como más de 35 especies y 21 familias.

Del análisis comparativo de los tres sitios, el bosque en regeneración presentó la mayor riqueza, abundancia y diversidad, seguido muy de cerca del bosque maduro y por último el bosque con cafetal. Estos resultados muy probablemente son producto de la heterogeneidad ambiental o de la estructura de la vegetación que presentan los sitios tanto horizontal como verticalmente (MacArthur \& MacArthur 1961, Nocedal 1984), ya que se ha sugerido que los bosques en regeneración natural (bosques secundarios), mantienen en ciertas épocas del año una dinámica mayor en cuanto a producción de semillas que los bosques maduros (Flores \& Dezzeo 2005), soportando de esta manera una gran diversidad de especies, en donde confluyen varios gremios alimentarios. Además, cuentan con más especialistas, más especies endémicas y mayor abundancia de insectívoros (Wunderle \& Latta 1996), así como de especies migratorias debido a la disponibilidad de nichos ecológicos (Hutto 1980). Trabajos similares donde las áreas en regeneración resultaron más diversas que los bosques maduros y asociados a alguna plantación son los de Blake y Loiselle (2001), Bojorges y López-Mata (2005) y Lentijo y Kattan (2005).

Es posible también que la mayor diversidad y abundancia encontrada en el bosque en regeneración, sea producto de que las áreas de bosque maduro y con cafetal presentan una mayor altura del dosel, lo que limitó una mayor captura y/u observación de los individuos que habitan en la parte alta del bosque (Verea et al. 2000), como por ejemplo muchas especies migratorias que suelen forrajear en el follaje denso del dosel más alto, lo que permite que las especies residentes se distribuyan en otros pisos de las áreas de bosque maduro y con cafetal, reduciendo de esta forma la competencia (Greenberg 1986).

De manera general y de acuerdo a las categorías de Pettingill (1969), en los tres sitios de estudio predominaron las especies raras sobre las abundantes y en promedio un $35 \%$ de las especies en los tres sitios de acuerdo a los números de Hill son especies no abundantes con registros de 3 a 1 individuos, que no contribuyen de manera efectiva a la diversidad de especies en los mismos. En las comunidades, existe una característica sui generis que incluye pocas especies abundantes y muchas raras (Krebs 2000). En este sentido, sólo la especie Myadestes occidentalis resultó ser abundante en los tres sitios de estudio, y las especies Amazilia beryllina, Buarremon brunneinucha y Chlorospingus ophthalmicus fueron abundantes en dos sitios.

De manera interesante, existen especies congenéricas como Basileuterus rufifrons, B. belli y B. culicivorus, así como Myioborus pictus y M. miniatus que 
resultaron ser abundantes cada una en un sitio diferente. Lo anterior sugiere que factores intrínsecos como las diferencias conductuales y su variación estacional juegan un papel dominante en la segregación y estructuración de las comunidades (Terborgh \& Weske 1975, Verea et al. 2000, Codesido \& Bilenca 2004), además de las relaciones de competencia. Las especies anteriormente mencionadas a pesar de ser hermanas y con hábitos similares en cuanto a su forrajeo, aparentemente poseen requerimientos específicos muy diferentes, con lo que posiblemente sus nichos no se sobrelapan, aunque esto último tendría que ser comprobado con la utilización de herramientas que modelan el nicho fundamental proyectado en un espacio ecológico y en una dimensión espacial mucho mayor (e.g. Nakazawa et al. 2004, Peterson \& Holt 2003).

Por otra parte, se ha sugerido también que la disponibilidad de recursos alimentarios es uno de los factores determinantes en la dinámica y estructuración de las comunidades de aves (Nocedal 1984, Wolda 1990, Smith \& Rotenberry 1990). Esta hipótesis parece ser explicada en este estudio, ya que el gremio de las aves insectívoras fue el componente más importante en los tres sitios de estudio con cerca del $50 \%$ del total de especies (Apéndice 1). Cabe resaltar que las especies insectívoras son de amplia distribución en América (Barden 1941). Otra de las razones que determinan este patrón de dominancia, es que éstas presentan variaciones conductuales y especializaciones morfológicas (Holmes 1990), existiendo especies que prefieren los bosques abiertos y secos (Hutto 1980, Anjos et al. 1997), o bien, bosques maduros o conservados con altos niveles de humedad, que mantienen en ciertas épocas del año un suministro relativamente constante de invertebrados (Ramírez-Albores 2006).

Las especies omnívoras también representaron una alta proporción en los tres sitios, muchas de las cuales prefieren los bosques secundarios (e.g. Crypturellus cinnamomeus, Aphelocoma ultramarina, Cyanocitta stelleri y Melanotis caerulescens). Los granívoros tuvieron mayor riqueza en el bosque en regeneración, debido a que en los alrededores de esta zona existen cultivos que proveen de granos a las aves (Columbina inca, C. passerina, Carduelis psaltria). Sin embargo, no todos los granívoros son indicadores de un pobre estado de conservación de los hábitats, ya que especies como Atlapetes pileatus, Buarremon brunneinucha, Pipilo ocai y Aimophila rufescens, que también consumen insectos, son especies que se alimentan de granos o semillas en condiciones naturales y que se les observó en zonas con buen estado de conservación.

La riqueza de los depredadores compuesta principalmente por carnívoros fue ligeramente mayor en el bosque maduro, donde se observaron algunas rapaces perchando generalmente en el dosel alto. Estas especies por estar en la cumbre de la pirámide alimentaria, son excelentes indicadores del buen estado de los hábitats, de ahí que este sitio haya tenido la mayor riqueza de carnívoros, ya que fue el que 
presentó el mejor estado de conservación. Los insectívoro-frugívoros fueron ligeramente más abundantes en el bosque maduro, ya que se benefician de la gran cantidad de insectos disponibles en la época de lluvias que se encuentran entre la corteza y el follaje denso de los árboles (Wolda 1990), complementando su dieta con algunos frutos de la zona particularmente de las especies Byrsonima crassifolia (nanche), Rubus sp. (zarzamora) y Prunus sp. (capulín) (Almazán-Núñez obs. pers.), así como de otras plantas con frutos carnosos que abundan en bosques con mayores condiciones de humedad (Poulin et al. 1994, Verea et al. 2000).

La riqueza de los nectarívoros en los tres sitios fue relativamente baja, probablemente debido a la escasa floración que se observó en la zona, la cual es marcadamente estacional (Arizmendi et al. 1990). Esto sugiere que las especies de este gremio diversifican sus hábitos alimentarios y consumen insectos como fuente de proteínas (Lara \& Ornelas 1998), además de que es sabido que algunas especies de este gremio realizan movimientos altitudinales resultado de la búsqueda de recursos (Almazán-Núñez \& Navarro 2006).

Diferentes estudios han destacado que la riqueza y la abundancia generalmente aumentan con la complejidad estructural del hábitat, lo cual a su vez incrementa el número de estratos y la disponibilidad de alimento (Pearson 1971, Nocedal 1984, González-Ortega \& Morales-Pérez 1998, Cueto \& López de Casenave 1999, Bojorges \& López-Mata 2001, Lentijo \& Kattan 2005). En los tres sitios de estudio, el estrato medio soportó una mayor cantidad de individuos. Esto es debido a que en éste confluyen especies de los estratos inferior y superior, lo cual sugiere que muchas de las especies registradas utilizan el estrato medio como una opción viable para el descanso, refugio y vigilancia (Nocedal 1984). Asimismo, otros estudios realizados en bosques templados del sur de México se han destacado por contener una alta proporción de especies en los estratos inferior o medio (e.g. González-Ortega 1998).

La distribución vertical de las especies puede ser explicada desde el punto de vista trófico. En el estrato medio confluyen varios gremios alimentarios como los insectívoros de follaje y los insectívoros acechadores (Arizmendi et al. 1990), los cuales son los más importantes en la zona de estudio en términos de riqueza y abundancia. El segundo estrato en importancia varió entre los sitios, siendo el estrato inferior para el bosque en regeneración y el estrato superior para el bosque maduro y con cafetal. En el bosque en regeneración, la cantidad de luz que penetra es mayor, dado que no existe un follaje muy denso y la mayoría de los árboles están en proceso de crecimiento, lo cual favorece el desarrollo de gramíneas que crecen simultáneamente con la mayoría de los árboles jóvenes. Es por esta razón, que la mayor cantidad de granívoros fueron registrados en este sitio, lo que apoya los resultados de Nocedal (1984), quién indicó que en los bosques de pino con gran cobertura de pastos los granívoros son dominantes. En el caso del bosque maduro y bosque con cafetal, la mayor cobertura en cuanto al follaje permitió el registro de una 
gran cantidad de individuos en las partes altas del dosel, especialmente de insectívoros del follaje, que forrajean en grupos grandes, como algunas especies de la familia Parulidae. Además, una posible razón para la disminución de aves en el estrato inferior del bosque con cafetal, es precisamente la transformación de este estrato por la sustitución de las plantas originales del sotobosque por la plantas de café (Perfecto et al. 1996).

Aún cuando la conservación y el manejo de las aves requiere de información detallada sobre sus parámetros de diversidad y abundancia (Remsen 1994), pocos son los estudios locales dentro del estado de Guerrero enfocados a medir la relación entre las aves y sus requerimientos de hábitat. Esta información, es crucial para el esclarecimiento de los procesos ecológicos de la comunidad de aves de esta región, así como para su aplicación en acciones prácticas para su conservación, más aún cuando los bosques montanos del centro de Guerrero han sido identificados desde el punto de vista avifaunístico como prioritarios para su conservación a nivel nacional (Álvarez \& Morrone 2004), debido a la alta riqueza y endemismo que presentan.

AGRADECIMIENTOS. Agradecemos a Luis A. Sánchez-González y Octavio Rojas-Soto, así como a dos revisores anónimos los valiosos comentarios hechos a una versión inicial del manuscrito. La participación de América Hinterholzer fue crucial en el desarrollo de este manuscrito. Oscar Nova, J. Carmen Mendoza, Guillermo T. Brito, Samuel García, Marcos Clemente y Elida Moreno colaboraron de manera decisiva en el trabajo de campo. Al Proyecto de Conservación de la Biodiversidad por Comunidades Indígenas (COINBIO) y PROCYMAF-CONAFOR, de donde se obtuvo el apoyo económico para la realización de este estudio.

\section{LITERATURA CITADA}

Almazán-Núñez, R. C. \& A. G. Navarro S. 2006. Avifauna de la subcuenca del río San Juan, Guerrero, México. Revista Mexicana de Biodiversidad. 77: 103-114

Álvarez, M. E. \& J. J. Morrone. 2004. Propuesta de áreas para la conservación de aves de México, empleando herramientas panbiogeográficas e índices de complementariedad. Interciencia. 29: 112-120.

American Ornithologist's Union (AOU). 1998. Check-list of North American birds. 7th edition. American Ornithologists' Union, Washington, D.C. USA.

Anjos, L. dos, K. L. Schuchmann \& R. Berndt. 1997. Avifaunal composition species richness and status in the Tibagi river basin, Parana state, southern Brazil. Ornitología Neotropical. 8: 145-174

Arizmendi, M. C., H. Berlanga, L. Márquez-Valdelamar, L. Navarijo \& F. Ornelas. 1990. Avifauna de la región de Chamela, Jalisco. Cuadernos 4. Instituto de Biología, UNAM. México, D.F.

Arnold, K. A. 1971. Three additional specimens of the Eared-poorwill from the state of Guerrero, Mexico. Condor. 73: 475.

Arnold, K. A. \& T. Maxwell. 1970. The Great Swallow-tailed Swift (Panyptyla sanctihieronymi) from the state of Guerrero, Mexico. Condor. 72: 108.

Balmer, O. 2001. Species lists in ecology and conservation: Abundances matter. Conservation Biology. 16: $1160-1161$.

Barden, A. A. 1941. Distribution of the families of birds. Auk. 58: 543-557

Blake, E. R. 1950. A report on a collection of birds from Guerrero, Mexico. Fieldiana Zoology. 31: 373 392 
Blake, J. G. \& B. A. Loiselle. 2001. Bird assemblages in second-growth and old-growth forest, Costa Rica: perspectives from mist nets and point counts. Auk. 118: 304-326

Bojorges, B. J. \& L. López-Mata. 2001. Abundancia y distribución temporal de aves en una selva mediana subperennifolia en el centro de Veracruz, México. Anales del Instituto de Biología UNAM, Serie Zoología. 72: 259-283

Bojorges, B. J. \& L. López-Mata. 2005. Riqueza y diversidad de especies de aves en una selva mediana subperennifolia en el centro de Veracruz, México. Acta Zoológica Mexicana (n.s.). 21: 1-20.

Brower, J. E., J. H. Zar \& C. N. von Ende. 1998. Field and laboratory methods for general ecology. Fourth Ed. McGraw-Hill. USA.

Challenger, A. 1998. Utilización y conservación de los ecosistemas terrestres de México: Pasado, presente y futuro. CONABIO-Instituto de Biología UNAM-Sierra Madre. México, D. F.

Chazdon, R. L., R. K. Colwell, J. S. Denslow \& M. Guariguata. 1998. Statistical estimation of species richness of woody regeneration in primary and secondary rainforest of northeastern Costa Rica. Pp. 185-309. In: F. Dallmeier \& J. Comisky (eds). Forest biodiversity in North, Central, and South America and the Caribbean: Research and monitoring. Parthenon, Paris.

Coates-Estrada, R. \& A. Estrada. 1985. Lista de las aves de la Estación de Biología Los Tuxtlas. Instituto de Biología, UNAM. México, D. F.

Codesido, M. \& D. Bilenca. 2004. Variación estacional de un ensamble de aves en un bosque subtropical semiárido del Chaco Argentino. Biotropica. 36: 544-554.

Colwell, R. K. 2006. EstimateS: Statistical estimation of species richness and shared species from samples. Version 8. User's guide and application published at: http://viceroy.eeb.uconn.edu/estimates

Colwell, R. K. \& J. Coddington. 1994. Estimating terrestrial biodiversity through extrapolation. Philosophical Transaction Royal Society Series B. 345: 101-118.

Cueto, V. R. \& J. Lopez de Casenave. 1999. Determinants of bird species richness: Role of climate and vegetation structure at a regional scale. Journal of Biogeography 26: 487-492.

Diario Oficial de la Federación (DOF). 2002. Norma Oficial Mexicana NOM-059-ECOL-2001, Protección ambiental-Especies nativas de México de flora y fauna silvestres-Categorías de riesgo y especificaciones para su inclusión, exclusión o cambio-Lista de especies en riesgo.

Dixon, K. L. \& W. B. Davis. 1958. Some additions to the avifauna of Guerrero, México. Condor. 60:407

Ehrlich, P. R., D.S. Dobkin \& D. Wheye. 1988. The Birder's handbook: A field guide to the natural history of North American birds. Simon and Schuster. New York, USA.

Emlen, J. M. 1971. Population densities of birds derived from transect counts. Auk. 88: 323-342.

Escalante, P., A. G. Navarro \& A. T. Peterson. 1998. Un análisis geográfico, ecológico e histórico de la diversidad de aves terrestres en México. Pp. 279-304. In: T. P. Ramamoorthy, R. Bye, A. Lot \& J. Fa (eds). Diversidad Biológica de México: Orígenes y distribución. Instituto de Biología, UNAM. México, D. F.

Flores, S. \& N. Dezzeo. 2005. Variaciones temporales en cantidad de semillas en el suelo y en lluvia de semillas en un gradiente bosque-sabana en la Gran Sabana, Venezuela. Interciencia. 30: 39-43.

Flores-Villela, O. \& P. Gérez. 1994. Biodiversidad y conservación en México: Vertebrados, vegetación y uso del suelo. CONABIO-UNAM, México, D. F.

Fregoso, A., A. Velázquez, G. Bocco \& G. Cortéz. 2001. El enfoque de paisaje en el manejo forestal de la comunidad indígena de Nuevo San Juan Parangaricutiro, Michoacán, México. Boletín del Instituto de Geografía UNAM 46: 58-77.

García, E. 1981. Modificaciones al sistema de clasificación climática de Copen (para adaptarlo a las condiciones de la República Mexicana). Offset Larios, México D. F. 
Gómez de Silva, H. \& R. Medellín. 2001. Evaluating completeness of species lists for conservation and macroecology: Case-study of Mexican land birds. Conservation Biology. 15: 1384-1395.

González-García, F. \& H. Gómez de Silva. 2003. Especies endémicas: riqueza, patrones de distribución y retos para su conservación. Pp. 150-194. In: H. Gómez de Silva \& A. Oliveras de Ita (eds). Conservación de aves: Experiencias en México. CIPAMEX, CONABIO, NFWF, México, D. F.

González-Ortega, M. A. \& J. E. Morales-Pérez. 1998. Distribución vertical de la avifauna en un bosque templado de Zinacantan, Chiapas, México. Acta Zoológica Mexicana 75: 125-142.

Griscom, L. 1937. A collection of birds from Omilteme, Guerrero. Auk. 54: 192-199.

Greenberg, R. 1986. Competition in migrant birds in the nonbreeding season. Pp. 281-303. In: R. F. Johnston (ed). Current Ornithology. Vol. 3. Plenum Press, New York.

Holmes, R. T. 1990. Ecological and evolutionary impacts of bird predation on forest insects: An overview. Pp. 6-13. In: M. L. Morrison, C. J. Ralph, J. Verner \& J. R. Jehl (eds). Avian foraging: Theory, methodology, and applications. Studies in Avian Biology 13.

Howell, S. N. G. \& S. Webb. 1995. A guide to the birds of Mexico and Northern central America. Oxford University Press. New York, USA.

Hutto, R. L. 1980. Winter habitat distribution of migratory land birds in western Mexico, with special reference to small foliage-gleaning insectivores. Pp. 181-203. In: Keast \& Morton (eds). Migrant Birds in the Neotropics: Ecology, Behavior, Distribution and Conservation Washington, D.C. USA.

Krebs, C. J. 2000. Ecología: Estudio de la distribución y la abundancia. Segunda edición. Oxford University Press. México. D. F.

Lara, C. \& J. F. Ornelas. 1998. Forrajeo de artrópodos por dos colibríes mexicanos en condiciones de aviario. Ornitología Neotropical 9: 41-50.

Lentijo, G. M. \& G. Kattan. 2005. Estratificación vertical de las aves en una plantación monoespecífica y en un bosque nativo en la cordillera central de Colombia. Ornitología Colombiana. 3: 51-61

Longino, J. T. \& R. K. Colwell. 1997. Biodiversity assessment using structured inventory: capturing the ant fauna of a tropical rain forest. Ecological Applications. 7: 1263-1277.

Ludwig, J. A. \& J. F. Reynolds. 1988. Statistical ecology: A primer on Methods and computing. Jonh Wiley \& Sons. New York, USA.

Luna, I. \& J. Llorente (eds). 1993. Historia natural del parque ecológico estatal Omiltemi, Chilpancingo, Guerrero, México. CONABIO-UNAM. México, D. F.

MacArthur, R. H. \& W. MacArthur. 1961. On bird species diversity. Ecology. 42: 594-598.

Magurran, A. E. 1989. Diversidad ecológica y su medición. Primera edición. Ediciones Vedrá. Barcelona, España.

Martín del Campo, R. 1948. Contribución para el conocimiento de la fauna ornitológica del estado de Guerrero. Anales del Instituto de Biología UNAM, Serie Zoología. 19: 241-266.

Miller, A. H. \& R. W. Storer. 1950. A new race of Parus sclateri from the Sierra Madre del Sur, Mexico. J. Washington Academy Science. 40: 301-302.

Moreno, C. E. 2001. Métodos para medir la biodiversidad. M\&T - Manuales y Tesis SEA, vol 1. Zaragoza.

Nakazawa, Y., A. T. Peterson, E. Martínez-Meyer \& A. G. Navarro-Sigüenza. 2004. Seasonal niches of neartic-neotropical migratory birds: Implications for the evolution of migration. Auk. 121: 610-618.

National Geographic Society. 1999. Field guide to the birds of North America. National Geographic Society. Tercera Edición. Washington, D.C. USA

Navarro, A. G. 1998. Distribución geográfica y ecológica de la avifauna del estado de Guerrero, México. Tesis de Doctorado. Facultad de Ciencias, UNAM. México, D. F.

Navarro, A. G. \& M. Muñoz A. 1990. Aves, reptiles y anfibios del parque estatal Omiltemi, Chilpancingo, Guerrero. Pp 247-258 In: F. Rivera \& J. L. Camarillo (comps). Áreas protegidas en México y especies en extinción. ENEP Iztacala UNAM. México, D. F. 
Navarro, A. G. \& H. Benítez. 1993. Patrones de riqueza y endemismo de las aves. Revista Ciencias No. Esp. 7:45-54

Navarro, A. G. \& P. Escalante P. 1993. Aves. In: I. Luna y J. Llorente (eds). Historia natural del parque ecológico estatal Omiltemi, Chilpancingo, Guerrero, México. CONABIO-UNAM. México, D. F.

Nocedal, J. 1984. Estructura y utilización del follaje de las comunidades de pájaros en bosques templados del Valle de México. Acta Zoológica Mexicana. 6: 1-37.

Ojasti, J. 2000. Manejo de fauna silvestre neotropical. F. Dalmeier (ed.). SIMAB Series No. 5. Smithsonian Institution/MAB Program, Washington, D.C.

Pearson, D. L. 1971. Vertical stratification of birds in a tropical dry forest. Condor. 73: 46-55.

Perfecto, I., R. Rice, R. Greenberg \& M. Van der Voort.1996. Shadde coffe: A disappearing refuge for biodiversity. BioScience. 46: 598-608.

Peterson, A. T. \& R. D. Holt. 2003. Niche differentiation in Mexican birds: Using point occurrences to detect ecological innovation. Ecology Letters. 6: 774-782

Peterson, R. T. \& E. L. Chalif. 1989. Guía de campo de las Aves de México. Diana. México, D. F.

Pettingill, O. S. Jr. 1969. Ornithology in Laboratory and Field. Cuarta edición. Burgess, Minneapolis, Minnesota, USA.

Poulin, B., G. Lefebvre \& R. McNeil. 1994. Characteristics of feeding guilds and variation in diets of bird species of three tropical sites. Biotropica 26: 187-197

Puebla-Olivares, F. 2001. Aspectos ecológicos de la familia Dendrocolaptidae (Aves) en la localidad de Yaxchilán, Chiapas. Tesis de Maestría, Facultad de Ciencias, UNAM. México, D. F.

Ramírez-Albores, J. E. 2006. Variación en la composición de comunidades de aves en la Reserva de la Biosfera Montes Azules y áreas adyacentes, Chiapas, México. Biota Neotropica. 6: 1-19.

Remsen, J. V. 1994. Use and misuse of bird lists in community ecology and conservation. Auk 111: 225227.

Rzedowski, J. 1978. Vegetación de México. Limusa. México, D. F.

Smith, K. G. \& J. T. Rotenberry. 1990. Quantifying food resources in avian studies: Present problems and future needs. Pp. 3-5. In: M. L. Morrison, C. J. Ralph, J. Verner \& J. R. Jehl (eds). Avian foraging: Theory, methodology, and applications. Studies in Avian Biology 13.

Stiles, F. G., A. F. Skutch \& D. Gardner. 2003. Guía de aves de Costa Rica. Tercera edición, INBIO. Costa Rica.

Terborgh, J. \& J. Weske. 1975. The role of competition in the distribution of Andean birds. Ecology 56: 562-576.

Toledo, V. M. \& M. J. Ordóñez. 1998. El panorama de la biodiversidad de México: Una revisión de los hábitats terrestres. Pp. 739-757. In: T. P. Ramamoorthy, R. Bye, A. Lot \& J. Fa. (eds). Diversidad Biológica de México: Orígenes y distribución. Instituto de Biología, UNAM. México, D. F.

Van Rossem, A. J. 1940. A new race of the warbling vireo from Guerrero, Mexico. Transaction San Diego Society Natural History. 11: 77-78

Verea, C., A. Fernández-Badillo \& A. Solórzano. 2000. Variación en la composición de las comunidades de aves de sotobosque de dos bosques en el norte de Venezuela. Ornitología Neotropical. 11: 65-79.

Wolda, H. 1990. Food availability for an insectivore and how to measure it. Pp. 38-43. In: M. L. Morrison, C. J. Ralph, J. Verner \& J. R. Jehl (eds). Avian foraging: Theory, methodology, and applications. Studies in Avian Biology 13.

Wunderle, J. M. Jr. \& S. C. Latta. 1996. Avian abundance in sun and shade coffee plantation and remnant pine forest in the cordillera Central, Dominican Republic. Ornitología Neotropical. 7: 1934 


\section{Apéndice 1}

Especies registradas en bosques de pino-encino del centro de Guerrero, México. La secuencia y el arreglo sistemático sigue a la American Ornithologist's Union (AOU 1998). Abundancia: $\mathrm{A}=$ abundante, $\mathrm{C}=$ común, $\mathrm{MC}=$ moderadamente común, $\mathrm{NC}=$ no común, $\mathrm{R}=$ rara. ${ }^{1}$ y 2 especies abundantes y muy abundantes y que contribuyen a la diversidad de especies de acuerdo a N1 y N2 de Hill respectivamente. Estacionalidad: $\mathrm{RP}=$ residente permanente, $\mathrm{MI}=$ migratoria de invierno, $\mathrm{MV}=$ migratoria de verano. Dieta: $\mathrm{Ca}=$ carroñero $\mathrm{C}=$ carnívoro, $\mathrm{F}-\mathrm{I}=$ frugívoroinsectívoro, $\mathrm{G}=$ granívoro, $\mathrm{I}=$ insectívoro, $\mathrm{I}-\mathrm{C}=$ insectívoro-carnívoro, $\mathrm{I}-\mathrm{F}=$ insectívoro-frugívoro, $\mathrm{I}-\mathrm{G}=$ insectívoro-granívoro, $\mathrm{N}-\mathrm{I}=$ nectarívoro-insectívoro, $\mathrm{O}=$ omnívoro.

\begin{tabular}{|c|c|c|c|c|c|}
\hline \multirow[t]{2}{*}{ Especie } & \multicolumn{3}{|c|}{ Abundancia } & \multirow[t]{2}{*}{ Estacionalidad } & \multirow[t]{2}{*}{ Dieta } \\
\hline & regeneración & maduro & cafetal & & \\
\hline Crypturellus cinnamomeus & $\mathrm{R}$ & & & $\mathrm{RP}$ & $\mathrm{O}$ \\
\hline Coragyps atratus & $\mathrm{MC}^{1,2}$ & $\mathrm{R}$ & $\mathrm{MC}$ & $\mathrm{RP}$ & $\mathrm{Ca}$ \\
\hline Cathartes aura & $\mathrm{A}^{1,2}$ & $\mathrm{NC}^{1,2}$ & $\mathrm{C}^{1,2}$ & $\mathrm{RP}$ & $\mathrm{Ca}$ \\
\hline Accipiter striatus & & $\mathrm{NC} 1$ & & $\mathrm{RP}$ & $\mathrm{D}$ \\
\hline Accipiter cooperii & $\mathrm{R}$ & & & MI & $\mathrm{D}$ \\
\hline Buteo jamaicensis & $\mathrm{R}$ & $\mathrm{R}$ & & $\mathrm{RP}$ & $\mathrm{D}$ \\
\hline Falco sparverius & & $\mathrm{NC}^{1}$ & & MI & $\mathrm{D}$ \\
\hline Columbina inca & $\mathrm{A}^{1,2}$ & $\mathrm{NC}^{1,2}$ & & $\mathrm{RP}$ & G \\
\hline Columbina passerina & $\mathrm{NC} 1,2$ & & & $\mathrm{RP}$ & G \\
\hline Geotrygon albifacies & & & $\mathrm{NC}^{1,2}$ & $\mathrm{RP}$ & G \\
\hline Aratinga canicularis & & & $\mathrm{A}^{1,2}$ & $\mathrm{RP}$ & G \\
\hline Piaya cayana & $\mathrm{R}$ & & & $\mathrm{RP}$ & I \\
\hline Tyto alba & & $\mathrm{R}$ & & $\mathrm{RP}$ & $\mathrm{D}$ \\
\hline Glaucidium brasilianum & & & $\mathrm{R}$ & $\mathrm{RP}$ & $\mathrm{D}$ \\
\hline Ciccaba virgata & R1 & $\mathrm{R}^{1}$ & $\mathrm{R}^{1}$ & $\mathrm{RP}$ & $\mathrm{D}$ \\
\hline Chordeiles minor & R1 & & & MV & I \\
\hline Caprimulgus vociferus & & $\mathrm{R}^{1}$ & & $\mathrm{RP}$ & I \\
\hline Streptoprocne semicollaris & $\mathrm{C}^{1,2}$ & & & $\mathrm{RP}$ & I \\
\hline Phaetornis longirostris & & & $\mathrm{C}^{1,2}$ & $\mathrm{RP}$ & N-I \\
\hline Hylocharis leucotis & $\mathrm{MC}^{1,2}$ & $\mathrm{NC}^{1,2}$ & & $\mathrm{RP}$ & N-I \\
\hline Amazilia beryllina & $\mathrm{A}^{1,2}$ & $\mathrm{~A}^{1,2}$ & $\mathrm{C}^{1,2}$ & $\mathrm{RP}$ & N-I \\
\hline Eupherusa poliocerca & & & $\mathrm{MC}^{1,2}$ & $\mathrm{RP}$ & N-I \\
\hline Lampornis amethystinus & & $\mathrm{C}^{1,2}$ & & $\mathrm{RP}$ & N-I \\
\hline Lamprolaima rhami & & & $\mathrm{R}$ & $\mathrm{RP}$ & N-I \\
\hline Eugenes fulgens & $\mathrm{R}^{1}$ & $\mathrm{NC}^{1}$ & & $\mathrm{RP}$ & N-I \\
\hline Trogon mexicanus & $\mathrm{R}$ & $\mathrm{NC}^{1}$ & & $\mathrm{RP}$ & I-F \\
\hline Trogon collaris & & & $\mathrm{R}$ & $\mathrm{RP}$ & $\mathrm{I}-\mathrm{F}$ \\
\hline Momotus mexicanus & & & $\mathrm{R}$ & $\mathrm{RP}$ & $\mathrm{O}$ \\
\hline Aulacorhynchus prasinus & & $\mathrm{R}$ & $\mathrm{C}^{1,2}$ & $\mathrm{RP}$ & $\mathrm{O}$ \\
\hline Melanerpes formicivorus & $\mathrm{A}^{1,2}$ & $\mathrm{NC} 1,2$ & & $\mathrm{RP}$ & $\mathrm{I}-\mathrm{F}$ \\
\hline Picoides scalaris & & $\mathrm{NC}^{1,2}$ & & $\mathrm{RP}$ & $\mathrm{I}-\mathrm{F}$ \\
\hline Picoides villosus & $\mathrm{R}$ & $\mathrm{NC}^{1}$ & & $\mathrm{RP}$ & I \\
\hline Piculus auricularis & & & $\mathrm{NC}^{1}$ & $\mathrm{RP}$ & I \\
\hline Colaptes auratus & $\mathrm{R}$ & & & $\mathrm{RP}$ & I \\
\hline Anabacerthia variegaticeps & & & $\mathrm{R}$ & $\mathrm{RP}$ & I \\
\hline
\end{tabular}




\begin{tabular}{|c|c|c|c|c|c|}
\hline Especie & regeneración & $\begin{array}{c}\text { Abundancia } \\
\text { maduro }\end{array}$ & cafetal & Estacionalidad & Dieta \\
\hline Automolus rubiginosus & & & $\mathrm{NC}^{1,2}$ & $\mathrm{RP}$ & I \\
\hline Sittasomus griseicapillus & $\mathrm{R}$ & $\mathrm{R}$ & $\mathrm{NC} 1,2$ & $\mathrm{RP}$ & I \\
\hline Dendrocolaptes sanctithoma & & & $\mathrm{NC} 1$ & $\mathrm{RP}$ & I \\
\hline Xiphorhynchus flavigaster & & & $\mathrm{R}$ & $\mathrm{RP}$ & I \\
\hline Lepidocolaptes affinis & $\mathrm{R}$ & $\mathrm{NC}^{1,2}$ & & $\mathrm{RP}$ & I \\
\hline Myiopagis viridicata & & $\mathrm{MC}^{1,2}$ & & $\mathrm{RP}$ & I \\
\hline Rhynchocyclus brevirostris & & & $\mathrm{NC} 1,2$ & $\mathrm{RP}$ & I \\
\hline Mitrephanes phaeocercus & $\mathrm{MC}^{1,2}$ & $\mathrm{R}$ & $\mathrm{R}$ & $\mathrm{RP}$ & I \\
\hline Contopus pertinax & $\mathrm{MC}^{1,2}$ & $\mathrm{MC}^{1,2}$ & $\mathrm{R}^{1}$ & $\mathrm{RP}$ & I \\
\hline Empidonax affinis & $\mathrm{R}$ & $\mathrm{R}$ & $\mathrm{R} 1$ & $\mathrm{RP}$ & I \\
\hline Empidonax fulvifrons & $\mathrm{NC}^{1,2}$ & $\mathrm{R}$ & & $\mathrm{RP}$ & I \\
\hline Myiarchus tuberculifer & $\mathrm{NC}^{1,2}$ & $\mathrm{MC}^{1,2}$ & & $\mathrm{RP}$ & I \\
\hline Tyrannus vociferans & $\mathrm{R}$ & & & $\mathrm{RP}$ & I \\
\hline Pachyramphus aglaiae & $\mathrm{R}$ & & & $\mathrm{RP}$ & $\mathrm{I}-\mathrm{F}$ \\
\hline Tityra semifasciata & & & $\mathrm{MC}^{1,2}$ & $\mathrm{RP}$ & $\mathrm{I}-\mathrm{F}$ \\
\hline Vireo brevipennis & & $\mathrm{R}$ & & $\mathrm{RP}$ & I \\
\hline Vireo solitarius & $\mathrm{R}^{1}$ & & & MI & I \\
\hline Vireolanius melitophrys & $\mathrm{MC}^{1,2}$ & & & $\mathrm{RP}$ & I \\
\hline Cyanocitta stelleri & $\mathrm{NC}^{1}$ & & & $\mathrm{RP}$ & $\mathrm{O}$ \\
\hline Cyanocorax yncas & & & $\mathrm{NC}^{1,2}$ & $\mathrm{RP}$ & $\mathrm{O}$ \\
\hline Cyanolyca mirabilis & & $\mathrm{C}^{1,2}$ & & $\mathrm{RP}$ & $\mathrm{O}$ \\
\hline Aphelocoma californica & $\mathrm{R}$ & & & $\mathrm{RP}$ & $\mathrm{O}$ \\
\hline Aphelocoma ultramarina & $\mathrm{NC}^{1,2}$ & & & $\mathrm{RP}$ & $\mathrm{O}$ \\
\hline Corvus corax & $\mathrm{NC}^{1,2}$ & & & $\mathrm{RP}$ & $\mathrm{O}$ \\
\hline Stelgydopteryx serripenis & $\mathrm{C}^{1,2}$ & $\mathrm{~A}^{1,2}$ & & $\mathrm{RP}$ & I \\
\hline Hirundo rustica & & $\mathrm{NC}^{1,2}$ & & $\mathrm{RP}$ & I \\
\hline Psaltriparus minimus & $\mathrm{NC}^{1,2}$ & & & $\mathrm{RP}$ & I \\
\hline Certhia americana & $\mathrm{R} 1$ & $\mathrm{R}$ & & $\mathrm{RP}$ & I \\
\hline Thryothorus sinaloa & & & $\mathrm{MC}^{1,2}$ & $\mathrm{RP}$ & I \\
\hline Thryothorus pleurostictus & $\mathrm{NC}^{1,2}$ & & & $\mathrm{RP}$ & I \\
\hline Thryothorus felix & $\mathrm{R}^{1}$ & & & $\mathrm{RP}$ & I \\
\hline Troglodytes aedon & & $\mathrm{R}$ & & $\mathrm{RP}$ & I \\
\hline Henicorhina leucophrys & & $\mathrm{A}^{1,2}$ & $\mathrm{C}^{1,2}$ & $\mathrm{RP}$ & I \\
\hline Regulus calendula & $\mathrm{R}$ & & & MI & I \\
\hline Polioptila caerulea & $\mathrm{C}^{1,2}$ & & & MI & I \\
\hline Sialia sialis & $\mathrm{C}^{1,2}$ & $\mathrm{MC}^{1,2}$ & & $\mathrm{RP}$ & $\mathrm{I}-\mathrm{F}$ \\
\hline Myadestes occidentalis & $\mathrm{A}^{1,2}$ & $\mathrm{~A}^{1,2}$ & $\mathrm{~A}^{1,2}$ & $\mathrm{RP}$ & $\mathrm{I}-\mathrm{F}$ \\
\hline Catharus aurantiirostris & $\mathrm{NC}^{1,2}$ & $\mathrm{NC}^{1,2}$ & $\mathrm{MC}^{1,2}$ & $\mathrm{RP}$ & $\mathrm{I}-\mathrm{F}$ \\
\hline Catharus occidentalis & & $\mathrm{C}^{1,2}$ & & $\mathrm{RP}$ & $\mathrm{I}-\mathrm{F}$ \\
\hline Catharus frantzii & & $\mathrm{R}^{1}$ & & $\mathrm{RP}$ & $\mathrm{I}-\mathrm{F}$ \\
\hline Turdus assimilis & $\mathrm{MC}^{1,2}$ & $\mathrm{R}$ & $\mathrm{C}^{1,2}$ & $\mathrm{RP}$ & $\mathrm{I}-\mathrm{F}$ \\
\hline Turdus migratorius & $\mathrm{R}^{1}$ & $\mathrm{C}^{1,2}$ & & $\mathrm{RP}$ & $\mathrm{I}-\mathrm{F}$ \\
\hline Melanotis caerulescens & $\mathrm{MC}^{1,2}$ & $\mathrm{NC} 1,2$ & & $\mathrm{RP}$ & $\mathrm{O}$ \\
\hline Ptilogonys cinereus & $\mathrm{MC}^{1,2}$ & $\mathrm{NC}^{1,2}$ & & $\mathrm{RP}$ & $\mathrm{I}-\mathrm{F}$ \\
\hline Vermivora celata & $\mathrm{R}$ & & & MI & I \\
\hline Vermivora ruficapilla & & $\mathrm{C}^{1,2}$ & & MI & I \\
\hline Vermivora crissalis & & $\mathrm{R}$ & & $\mathrm{RP}$ & I \\
\hline Parula superciliosa & & & $\mathrm{R}$ & $\mathrm{RP}$ & $\mathrm{I}$ \\
\hline
\end{tabular}


Almazán-Núñez et al.: Diversidad de aves en bosques de pino-encino de Guerrero

\begin{tabular}{|c|c|c|c|c|c|}
\hline Especie & regeneración & $\begin{array}{l}\text { Abundancia } \\
\text { maduro }\end{array}$ & cafetal & Estacionalidad & Dieta \\
\hline Dendroica coronata & $\mathrm{NC}^{1,2}$ & & & MI & I \\
\hline Dendroica nigrescens & $\mathrm{NC} 1$ & $\mathrm{MC}^{1,2}$ & & MI & I \\
\hline Dendroica townsendi & $\mathrm{NC}^{1,2}$ & $\mathrm{C}^{1,2}$ & & MI & I \\
\hline Dendroica occidentalis & & $\mathrm{R}$ & & MI & I \\
\hline Mniotilta varia & $\mathrm{C}^{1,2}$ & & $\mathrm{NC}^{1,2}$ & MI & I \\
\hline Seiurus motacilla & & & $\mathrm{R}$ & MI & I \\
\hline Oporornis tolmiei & & $\mathrm{R}$ & & MI & I \\
\hline Wilsonia pusilla & $\mathrm{NC}^{1,2}$ & $\mathrm{NC}^{1}$ & & MI & I \\
\hline Cardellina rubifrons & $\mathrm{R}$ & & & MI & I \\
\hline Ergaticus ruber & & $\mathrm{NC} 1,2$ & & $\mathrm{RP}$ & I \\
\hline Myioborus pictus & $\mathrm{A}^{1,2}$ & & & $\mathrm{RP}$ & I \\
\hline Myioborus miniatus & $\mathrm{C}^{1,2}$ & $\mathrm{~A}^{1,2}$ & & $\mathrm{RP}$ & I \\
\hline Basileuterus culicivorus & $\mathrm{NC}^{1,2}$ & & $\mathrm{~A}^{1,2}$ & $\mathrm{RP}$ & I \\
\hline Basileuterus rufifrons & $\mathrm{A}^{1,2}$ & & & $\mathrm{RP}$ & I \\
\hline Basileuterus belli & & $\mathrm{A}^{1,2}$ & & $\mathrm{RP}$ & I \\
\hline Chlorospingus ophthalmicus & & $\mathrm{A}^{1,2}$ & $\mathrm{~A}^{1,2}$ & $\mathrm{RP}$ & I \\
\hline Piranga flava & $\mathrm{C}^{1,2}$ & $\mathrm{MC}^{1,2}$ & & $\mathrm{RP}$ & $\mathrm{I}-\mathrm{F}$ \\
\hline Piranga bidentata & & & $\mathrm{R}$ & $\mathrm{RP}$ & $\mathrm{I}-\mathrm{F}$ \\
\hline Piranga erythrocephala & & & $\mathrm{R}$ & $\mathrm{RP}$ & $\mathrm{I}-\mathrm{F}$ \\
\hline Sporophila torqueola & & $\mathrm{R}$ & & $\mathrm{RP}$ & $\mathrm{G}$ \\
\hline Diglossa baritula & $\mathrm{R}$ & $\mathrm{MC}^{1,2}$ & & $\mathrm{RP}$ & N-I \\
\hline Atlapetes pileatus & $\mathrm{MC}^{1,2}$ & $\mathrm{NC}^{1,2}$ & & $\mathrm{RP}$ & G-I \\
\hline Buarremon brunneinucha & $\mathrm{MC}^{1,2}$ & $\mathrm{~A}^{1,2}$ & $\mathrm{~A}^{1,2}$ & $\mathrm{RP}$ & G-I \\
\hline Melozone kieneri & $\mathrm{MC} 1,2$ & $\mathrm{NC}^{1}$ & & $\mathrm{RP}$ & G-I \\
\hline Pipilo ocai & & $\mathrm{NC}^{1}$ & & $\mathrm{RP}$ & G-I \\
\hline Aimophila rufescens & $\mathrm{C}^{1,2}$ & & & $\mathrm{RP}$ & G-I \\
\hline Junco phaeonotus & $\mathrm{A}^{1,2}$ & $\mathrm{C}^{1,2}$ & & $\mathrm{RP}$ & G-I \\
\hline Pheucticus melanocephalus & $\mathrm{R}$ & $\mathrm{R}$ & & $\mathrm{RP}$ & $\mathrm{O}$ \\
\hline Passeina cyanea & & $\mathrm{R}$ & & MI & G-I \\
\hline Icterus wagleri & $\mathrm{NC}^{1,2}$ & & & $\mathrm{RP}$ & $\mathrm{O}$ \\
\hline Icterus graduacauda & $\mathrm{R}^{1}$ & $\mathrm{R}$ & $\mathrm{R}$ & $\mathrm{RP}$ & $\mathrm{O}$ \\
\hline Carpodacus mexicanus & $\mathrm{R}^{1}$ & & & $\mathrm{RP}$ & G \\
\hline Carduelis notata & $\mathrm{MC}^{1,2}$ & $\mathrm{C}^{1,2}$ & $\mathrm{C}^{1,2}$ & $\mathrm{RP}$ & G \\
\hline Carduelis psaltria & $\mathrm{NC}^{1,2}$ & & & $\mathrm{RP}$ & $\mathrm{G}$ \\
\hline
\end{tabular}

Recibido: 1 de marzo de 2008

Aceptado: 5 de septiembre de 2008 